\title{
El turismo como instrumento de colonización neoliberal: una perspectiva foucaultiana
}

\author{
Tourism as an instrument of neoliberal colonization: \\ a Foucaultian perspective
}

\author{
TZINTLI Chávez Luna \\ Universidad Autonóma \\ del Estado de México
}

\begin{abstract}
as realidades sociales son más complejas que los modelos que las representan, y el Lcaso del modelo de desarrollo no es la excepción. Así pues, las ideas sobre crecimiento, intervencionismo y modelos económicos, se reúnen en teorías parciales incapaces de describir la complejidad de la realidad; con lo que el concepto de desarrollo queda en la indefinición. Como consecuencia, tampoco es claro en qué se basa la diferenciación de países y regiones como desarrollados, subdesarrollados, o en desarrollo, tipología usada ampliamente en discursos académicos y políticos.
\end{abstract}

Es por ello que se observan situaciones que encierran contradicciones en el concepto (o modelo) de desarrollo y en su aplicación; y también se observa una falta de enfoques integrales que contemplen múltiples dimensiones y que sean congruentes con la historia, cultura, saberes y organización político-social de las comunidades. Esto ilustra que los discursos del desarrollo sean narrativas universalistas insostenibles, cuyos principios difícilmente resisten los cuestionamientos de la posmodernidad, que contradicen su explicatividad (Schuurman, 1993).

Con todo, a nivel internacional se atribuye al turismo un potencial de desarrollo; por lo que el "desarrollo turístico" se une frecuentemente a los países menos desarrollados, los cuales son extremadamente importantes como destinos y actores de la actividad global (Timothy \& Nyaupane, 2009, p. 6), pues además el turismo se considera como la única industria en el sector de servicios en la que los países en desarrollo (PED) e incluso los países más pobres, obtienen un saldo positivo en su balanza comercial con respecto a los países desarrollados (PD) (Epler Wood, 2007, p. 159).

En contraposición, se han subrayado los efectos negativos, como costos sociales y ambientales mayores que los beneficios (Nash, 1981). Además, en un contexto global el turismo tiene el riesgo de potencializar malentendidos, y probablemente su resultado no sea la paz y el entendimiento, sino el conflicto (Reisinger et al., 2013, p. 47). Y si bien pudiera suponerse que funde significados al mezclar individuos, culturas o naciones, bien puede resultar en interacciones complejas, difíciles de comprender.

A lo largo de esta crítica se explorarán algunos aspectos turísticos en torno a los discursos de desarrollo y a las otredades que de ellos surgen. Para lograrlo, se ha recurrido a ciertas nociones foucaultianas como discurso, juegos de verdad, significado histórico, agentes 
de normalización, panoptismo, dominación, micropoder, cuidado de sí y gubernamentalidad, con las cuales se explorarán algunas dinámicas de poder presentes entre los Otros involucrados en los discursos de turismo y desarrollo. El objetivo es ofrecer perspectivas que puedan resultar enriquecedoras para otros estudios y que promuevan un ejercicio de reflexión respecto de los discursos involucrados.

Cabe destacar que la presente crítica se realiza desde una posición que puede considerarse de Otredad desde distintos puntos de vista: la que escribe es una mujer situada en un contexto económico calificado como "en desarrollo" y con pertenencia socio-cultural a Latinoamérica (una de las periferias poscoloniales con respecto a Occidente), entre otras; por lo que representa una contraposición a las nociones unívocas de construcción de discursos históricos y sociales (Smith [1987, 1993] cit. por Humberstone, 2004, p. 130-131).

\section{Desarrollo y turismo}

El pensamiento occidental contiene un cúmulo de antagonismos entre los Otros; binomios lingüísticos que afirman a Uno en relación al Otro, negándole a la diferencia su entidad, autonomía, preexistencia y originalidad, y subyugando a la Otredad en una relación centro/ satélite (Ankor \& Wearing, 2013, p. 184-185). Entonces, los discursos están formados por parejas cuyos elementos estando en un mismo plano funcionan, sin embargo, como dos polos aparentemente irreconciliables. Este asunto es fundamental, porque la organización de los significantes en binomios privilegia siempre a un término, separa sus características y relega todo aquello que no es, lo que convierte al segundo término en el Otro, lo excluido, alguien cuyas características (físicas, sociales, culturales, cognitivas) niegan y desmienten las propias, conjunto de diferencias cuya naturaleza infinita implica la imposibilidad de reducción o exclusión absoluta (Derrida, 1994; 1996; 1998). El resultado es que la construcción de opuestos binarios los hace dependientes uno del otro (Hall \& Tucker [2004, p. 8] cit. por Cater, 2007, p. 57), y se crea una relación agónica entre los opuestos. De ahí lo irreconciliable entre Unos y Otros, construido y reafirmado históricamente, y la crítica a esa historia que afirma que el Otro se encuentra al margen del discurso, en un lugar más allá de los límites del lenguaje, que sin embargo es real.

Como parte de los discursos nacidos en Occidente, el del desarrollo se basa en construcciones binomiales, que de manera general se refieren a los opuestos Nosotros, normal, correcto/los Otros, anormal, desviado. Esta construcción no deja espacio a la voz de los Otros, que según el discurso, deben ser excluidos o disciplinados; y que al identificarse con IOS PED, concentran la Otredad en relación con los PD. Es decir, que se puede considerar al concepto de desarrollo como un juego de verdad a través del cual se moldea la imagen Propia y la de los Otros. De esta manera, los condicionamientos del ejercicio del poder moldean las percepciones de la gente, las regulan, organizan y autorizan institucionalmente, y por lo tanto, reflejan los discursos particulares sobre la Otredad, el turismo (Morrison, 2013, pp. 34 y 44) y el desarrollo. 
En la medida en que el Estado y las élites nacionales intervienen en la definición y orientación de las políticas de desarrollo, puede hablarse de gubernamentalidad, y por lo tanto, del desarrollo como tecnología de gobierno. Además del Estado, hay instituciones especializadas en las técnicas de gubernamentalidad en todos los niveles; su intervención se exacerbó con el neoliberalismo, con el que también las empresas han obtenido poder en el puro sentido foucaultiano de conducción de conductas.

Sin embargo, parece claro que los objetivos de las instituciones globales no pueden homologarse ni en diseño ni en aplicación para PD y PED, obligándonos a cuestionar su significado en la reconfiguración espacial, económica, sociocultural y política que acompaña a la globalización. Esto abre la interrogante de por qué se insiste en modelos de desarrollo cuya incompatibilidad con múltiples regiones en loS PED está tan extensamente documentada. Shuurman (1993), por ejemplo, relaciona el desarrollo con una gubernamentalidad institucional que actúa local y globalmente, y genera interacciones internacionales que no han podido ser explicadas ni resueltas, por lo que pese a su horizontalidad en el discurso, existen desigualdades que no se llegan a corregir. De aquí las crecientes diferencias socioeconómicas entre países ricos y pobres, pues los PED aplican políticas de corto plazo vinculadas a su deuda externa y no a largo y mediano plazos, mientras el crecimiento económico ha tenido impactos críticos en materia ambiental.

Debido a lo anterior, es válido preguntarse si los PED se ven obligados a aceptar los modelos respaldados o reconocidos por esas organizaciones o instituciones, a fin de obtener fondos para implementar sus políticas; agotando su resistencia y las oportunidades de supervivencia que pudieran tener a través de otras formas de economía. Entre los grupos sociales o las sociedades nacionales, la aceptación puede ser un síntoma de algo más sutil: que el discurso en cuestión se ha difundido tan eficientemente, que ellos mismos funcionan como agentes normalizadores. Así, lOS PED y sus individuos difícilmente se cuestionan si dichos discursos son compatibles con las conceptualizaciones locales de desarrollo, pobreza y riqueza; de forma que los actores involucrados y hasta los países mismos terminan asumiendo la necesidad de políticas para las cuales no tienen recursos financieros, por lo que recurren a instituciones que aseguran su adscripción al discurso.

Estas incongruencias son las mismas que permean gran parte de las relaciones basadas en la retórica del desarrollo occidental y que convierten a conceptos como el turismo y la sostenibilidad en discursos intervencionistas (Schuurman, 1993). Considerar esta perspectiva permite comprender por qué se plantea el empleo del turismo como herramienta de desarrollo, como la imposición de un dispositivo discursivo. Gracias a instrumentos de este tipo, el turismo se vuelve actividad económica importante para las naciones, mientras apoya la conservación ambiental y ofrecen a los pueblos tradicionales una oportunidad de "subir la escalera de la economía globalizada" (Epler Wood, 2007, p. 172). Pero esa "oportunidad" -asumida incluso académicamente- nació del discurso capitalista; y aunque inicialmente no sea compartida por Otras sociedades, podrá convertirse en su aspiración si los discursos se convierten en juegos de verdad y tecnologías de poder. Así, el turismo forma parte de las tecnologías que aseguran la continuidad del poder de las élites. 
Esto se nota cuando los objetivos de muchos proyectos de desarrollo se encuentran velados entre el discurso de un supuesto desarrollo que mejoraría la calidad de vida de los individuos de los PED. De esta forma se establecen relaciones ambiguas y confusas entre conceptos como desarrollo y pobreza; pero sobre todo porque no reconocen que la pobreza es una necesidad del modelo económico neoliberal, y que ha sido institucionalizada por los discursos y las tecnologías del desarrollo para conservar el estatus de dominación de los PD sobre los recursos económicos del planeta. Pareciera que en realidad se propone que los PED ofrezcan esos recursos a lOS PD a través de actividades terciarias, con la suposición de que las empresas serán sostenibles, o de que los pueblos desean ese tipo de desarrollo. Así, la relación entre los PED y los PD sería típicamente colonialista, pues existe el riesgo de que sus empresas controlen el uso de los recursos.

Así pues, ya que muchos programas y proyectos turísticos para combatir la pobreza son solo paliativos no adaptados a las realidades de las comunidades de los PED, no es sorprendente que las sociedades locales se desilusionen del turismo por la incapacidad que ha demostrado para cumplir con los beneficios locales que se prometen (cfr. Cater, 2007). De esta forma, las comunidades que reciben a los turistas sufren la dominación de discursos político-económicos que han resultado de la sustitución de conocimientos tradicionales por aquellos provenientes de una economía neoliberal globalizada. A través de estos discursos se ha creado en las comunidades una pobreza que favorece el flujo de recursos físicos y humanos al mercado global. Así, la clase dominante mantiene enclaves de mano de obra relativamente barata y motivos suficientes para la generación de campañas que promuevan una imagen socialmente aceptada de promoción del desarrollo.

De esta manera, el turismo puede verse como una forma de neocolonialismo, como parte de una gubernamentalidad global, y un vehículo de distribución de la política y doctrina neoliberal cuyos impactos, límites y gestión son distintos en PD y en PED (Timothy \& Nyaupane, 2009, p. 3). La actividad turística como tecnología de gobierno introduce conocimientos, procedimientos, valores y criterios para deslindar entre normal/anómalo; implica modificaciones en los usos del espacio, actividades productivas y costumbres, de modo que la gente presencia la sustitución de la economía tradicional, la extinción de los oficios, la emigración de residentes, la conversión de sus senderos en carreteras, la transformación de sus zonas de pesca en playas privadas, o la implantación de centros comerciales en sus viejos mercados.

Por eso es preciso considerar las profundas asimetrías que se infiltran en los encuentros turísticos, a través de mecanismos de control y dominación que han prevalecido por siglos, y que impulsan a los receptores a aceptar determinadas condiciones en su trato con el exterior, muchas veces quedando reducidos a la imposibilidad de oponerse, opinar, cuestionar o tomar decisiones, pues la construcción del Tercer Mundo, el subdesarrollo, los países "pobres" o los PED, es resultado de una compleja relación entre poder y conocimiento, que opera a escala local, regional, nacional y mundial.

Es importante señalar que en muchos casos la adopción del turismo como una nueva actividad productiva en una comunidad de un PED, depende en parte de la precariedad de 
su situación, porque muchos pueblos pueden admitirlo por una pobreza realmente grave, o por haber sufrido un muy alto grado de exclusión o saqueo de sus recursos. Esto podría significar que el grupo social en cuestión se ve obligado a someterse al ofrecimiento de grandes corporaciones, su propio Estado o cualquier otra organización relacionada, sin poder negociar mejores salarios o condiciones laborales, y mucho menos que se cumplan leyes ambientales o fiscales, por ejemplo, tal vez del mismo modo que en el pasado.

Si el grupo social quedara convencido del discurso - lo cual requiere cierto grado de normalización que guía a los individuos a creer que su bienestar depende del desarrolloimplicaría que los individuos aceptan las categorías desarrollado/no-desarrollado, y que se asumen a sí mismos como parte de dicho binomio. Lo anterior implica que a los PED no se les ha permitido construir sus propias conceptualizaciones y modelos, por lo que no se ha podido imaginar una realidad más allá del discurso occidental de desarrollo. En el fondo, también podría significar que sus esfuerzos de "vivir mejor" sean moldeados por discursos extranjeros, perdiéndose y convirtiéndose en Otros para sí mismos.

\section{Conclusión}

Con respecto a las ideas sobre el desarrollo, es posible argumentar que las teorías de desarrollo basadas en metadiscursos enfrentan retos fundamentales, como la imposibilidad de que los modelos sean universalizados (Telfer, 2009, pp. 157-160). Tal universalidad engarzada en el dispositivo panóptico adecuado, puede no tener otro resultado que el totalitarismo y derivar en un abuso del poder; una situación en la que ese poder carece de legitimidad y se imponen a los Otros las propias “fantasías, apetitos y deseos", creando una relación perennemente disimétrica (Foucault, 1999, pp. 400-405). Así pues, tendrían que construirse opciones vinculadas a la historia, cultura, saberes, aspiraciones y organización político-social de cada sociedad.

En todo caso, parece evidente que las relaciones PD y PED se replican cuando sus individuos se encuentran en un contexto turístico; y que resultan disimétricas porque se ha promovido un reparto inequitativo de recursos en el que las regiones "desarrolladas" los obtienen en exceso, contrariamente a los Otros. Sin embargo, también es disimétrico porque el mismo discurso otorga facilidades ideológicas, legales e institucionales a quienes lo han creado, lo cual impide que otras regiones que pretenden aplicarlo obtengan resultados favorables. De la misma manera, la difusión del discurso en regiones "en desarrollo" y la ausencia de propuestas alternativas resultan en un escaso cuestionamiento de los instrumentos que de él surgen. Ante esto, es pertinente cuestionar si algún paradigma de desarrollo existente o por venir pudiera emanciparse de este complejo sistema coercitivo

Esto significa entonces que la situación de dominación discursiva no se modificará hasta que la parte coartada asuma la completa responsabilidad de su propia libertad; en la cual no solo se encuentran los PED, sino todos y cada uno de los individuos que se han visto alcanzados por los discursos de desarrollo, muchos de los cuales los han interiorizado profundamente. 
Por estas razones, el cuestionar dichos mecanismos requiere un verdadero esfuerzo reflexivo que implicaría enfrentarse con la posibilidad de dejar atrás ciertas autoconcepciones, y aceptar que se es sujeto de un continuo control y observación. Este cuestionamiento se dificulta en la medida en que los individuos se encuentran normalizados; más aún cuando no se trata solamente de un proceso de liberación discursiva, sino de una práctica de libertad (Foucault, 1999, p. 395), un cuestionamiento profundo de las propias convicciones que permita a los individuos asumirse como entes libres para ver al Otro claramente como ser humano. Si esto ocurre, entonces también parece posible darse cuenta de que los Otros están de la misma forma sujetos a mecanismos de gubernamentalidad iguales o similares, los cuales no han permitido imaginar una convivencia inclusiva, no contestada y sin prejuicios.

\section{Literatura citada}

Ankor, J., \& Wearing, S. (2013). Gaze, Encounter and Philosophies of Otherness. En O. Moufakkir, \& Y. Reisinger (Eds.), The Host Gaze in Global Tourism (pp. 179-190). Oxfordshire, Reino Unido: CAB Intenatinoal.

Cater, E. (2007). Ecotourism as a western construct. En J. Higham (Ed.), Critical Issues in Ecoturism: Understanding a complex tourism phenomenon (pp. 46-69). Oxford, Reino Unido: Elsevier Ltd.

Derrida, J. (1994). The Deconstruction of Actuality. Radical Pholosophy(68), 28-41.

Derrida, J. (1996). Remarks on Deconstruction and Pragmatism. En C. Mouffe (Ed.), Deconstruction and Pragmatism (S. Critchley, Trad., pp. 77-90). Londres, Reino Unido: Routledge.

Derrida, J. (1998). Of Grammatology. (G. Chakravorty, Trad.). Baltimore: Johns Hopkins University Press.

Epler Wood, M. (2007). The role of sustainable tourism in international development: prospects for economic growth, alleviation of poverty and environmental conservation. En J. Higham (Ed.), Critical Issues in Ecoturism: Understanding a complex tourism phenomenon (pp. 158-184). Oxford, Reino Unido: Butterworth-Heinemann.

Foucault, M. (1999). La ética del cuidado de sí como práctica de la libertad. En M. Foucault, Estética, ética y hermenéutica (Á. Gabilondo, Trad., pp. 393-416). Barcelona, España: Paidós.

Humberstone, B. (2004). Standpoint research: multiple versions of reality in tourism theorising and research. En J. Phillimore, \& L. Goodson (Eds.), Qualitative Research in Tourism: Ontologies, epistemologies and methodologies (págs. 119-136). Londres, Reino Unido: Routledge.

Morrison, I. A. (2013). The Thai Host Gaze: Alterity and the Governance of Visitors in Thailand. En O. Moufakkir, \& Y. Reisinger (Edits.), The Host Gaze in Global Tourism (pp. 33-46). Oxfordshire, Reino Unido: CAB International. 
Nash, D. (1981). Tourism as an Anthropological Subject (Comments and Reply). Current Anthropology, 22(5), 461-481.

Reisinger, Y., Kozak, M., \& Visser, E. (2013). Turkish Host Gaze at Russian Tourists: a Cultural Perspective. En O. Moufakkir, \& Y. Reisinger (Eds.), The Host Gaze in Global Tourism (pp. 47-66). Oxfordshire, Reino Unido: CAB Intenational.

Schuurman, F. J. (1993). Introduction: Development Theory in the 1990. En F. J. Schuurman, \& F. J. Schuurman (Ed.), Beyond the Impasse: New Directions in Development Theory (pp. 1-47). Londres, Reino Unido: Zed Books.

Telfer, D. J. (2009). Development Studies and Tourism. En T. Jamal, \& M. Robinson (Eds.), The SAGE Handbook of Tourism Studies (pps. 146-165). Londres, Reino Unido: SAGE Publications Ltd.

Timothy, D. J., \& Nyaupane, G. (2009). Introduction: Heritage tourism and the less-developed world. En D. J. Timothy, \& G. P. Nyaupane (Eds.), Cultural Heritage and Tourism in the Developing World. A regional perspective (pp. 3-19). Abingon, Reino Unido: Routledge. 